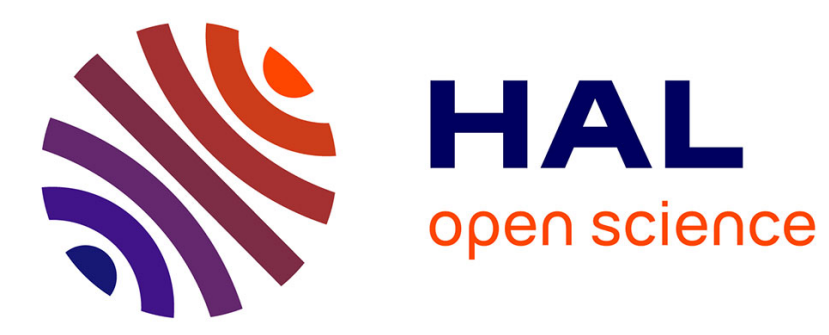

\title{
Decomposition of Low Rank Multi-Symmetric Tensor
} Jouhayna Harmouch, Bernard Mourrain, Houssam Khalil

\section{To cite this version:}

Jouhayna Harmouch, Bernard Mourrain, Houssam Khalil. Decomposition of Low Rank MultiSymmetric Tensor. MACIS 2017 - 7th International Conference on Mathematical Aspects of Computer and Information Sciences, Nov 2017, Vienna, Austria. pp.51-66, 10.1007/978-3-319-72453-9_4 . hal$01648747 \mathrm{v} 2$

\section{HAL Id: hal-01648747 \\ https://hal.inria.fr/hal-01648747v2}

Submitted on 30 Nov 2017

HAL is a multi-disciplinary open access archive for the deposit and dissemination of scientific research documents, whether they are published or not. The documents may come from teaching and research institutions in France or abroad, or from public or private research centers.
L'archive ouverte pluridisciplinaire HAL, est destinée au dépôt et à la diffusion de documents scientifiques de niveau recherche, publiés ou non, émanant des établissements d'enseignement et de recherche français ou étrangers, des laboratoires publics ou privés.

$$
\text { Copyright }
$$




\title{
Decomposition of Low Rank Multi-Symmetric Tensor
}

\author{
J. Harmouch ${ }^{1,2(凶)} \&$ B. Mourrain ${ }^{2(凶)} \&$ H. Khalil ${ }^{1}$ \\ 1 Laboratory of mathematics and its applications LaMa-Lebanon, \\ Lebanese University, Beirut, Lebanon \\ houssam.khalil@ul.edu.lb \\ 2 UCA, Inria, Aromath, Sophia Antipolis, France \\ \{jouhayna.harmouch,Bernard.Mourrain\}@inria.fr
}

\begin{abstract}
We study the decomposition of a multi-symmetric tensor $T$ as a sum of powers of product of linear forms in correlation with the decomposition of its dual $T^{*}$ as a weighted sum of evaluations. We use the properties of the associated Artinian Gorenstein Algebra $A_{\tau}$ to compute the decomposition of its dual $T^{*}$ which is defined via a formal power series $\tau$. We use the low rank decomposition of the Hankel operator $H_{\tau}$ associated to the symbol $\tau$ into a sum of indecomposable operators of low rank. A basis of $A_{\tau}$ is chosen such that the multiplication by some variables is possible. We compute the sub-coordinates of the evaluation points and their weights using the eigen-structure of multiplication matrices. The new algorithm that we propose works for small rank. We give a theoretical generalized approach of the method in $\mathrm{n}$ dimensional space. We show a numerical example of the decomposition of a multi-linear tensor of rank 3 in 3 dimensional space.
\end{abstract}

\section{Introduction}

The decomposition of symmetric and multi-symmetric tensors has many applications in engineering disciplines such that signal processing [11, scientific data analysis [9], 18, statistics [17, in bioinformatics and spectroscopy [5], in neuroscience, in phylogenetic .... For instance, the study of symmetric tensor decomposition gives an idea about the geometric structure of intersecting fibers in human brain using the Fibers Orientation Fibers Function described in [12], ou [8] and [19. The decomposition of multi symmetric tensors of small rank appear in several other contexts, for learning latent variable models which are algebraic statistics models. This is the case for the analysis of phylogenetic trees model described in [15] or for the analysis of contents of web pages model described in [1. Here, the mixture model is a collection of all non-negative probability tensors of low rank.

The tensor decomposition problem is also very interesting from an algebraic geometric point of view [10]. Important efforts have been developed over the last decades to better understand the theoretical aspects, as well as the algorithmic aspects of this difficult problem. Some of the well-known decomposition methods use local optimization techniques such Alternate Least Square, Gradient Descents, Quasi-Newton, ... to minimize the error between the tensor and its decomposition. Some other approaches exploit the algebraic structure associated to the tensor decomposition [4, [3. Homotopy techniques have also been used recently to compute such decomposition [2].

In this paper, we describe a direct method for the decomposition of multi-symmetric tensors, based on simple linear algebra tools. The decomposition algorithm applies to tensors of low enough rank. We follow the approach in [3] but directly apply numerically stable linear algebra tools on submatrices of the Hankel matrices to recover the decomposition. In particular, we show how to recover directly the points and weights from eigenvectors of multiplication operators of the quotient algebra associated to the decomposition. The algorithm does not require the solution of polynomial equations. The proposed method extends the techniques of [16] to more general tensors and to tensors of higher rank. It is closely connected to the multivariate Prony method investigated in [14] and to the structured low rank decomposition of Hankel matrix [7].

A multi-linear tensor is in correspondence with a multilinear map from a product of vector spaces to the coefficient field. A tensor symmetric tensor is a tensor whose components stay invariant by any permutation of indices. In the following, we study the general class of multi symmetric tensor decomposition problem, which 
contains these two classes. We show the correlation between the dual of a tensor, formal power series and then the Hankel matrices associated to them. We use the singular value decomposition of Hankel matrices to compute the decomposition of a tensor of low rank. We exploit the properties of Artinian Gorenstein Algebra to find out some multiplication matrices which help to know the eigen-structure of points associated to linear forms and their weights. We slice variables into bunches of sub-variables and we adapt the description of Artinian Gorenstein Algebra to this case. We adapt the method of decomposition of Hankel matrices of low rank described in [7] to a decomposition of multi linear tensors method which is based on the decomposition of a formal power series as a weighted sum of exponential described in [14]. The computation of multiplication matrices depend on the dimension of tensor, and the number of given moments or coefficients. We describe the algorithm in 3 dimensional space and we give its numerical implementation using MAPLE. This description gives an idea about the constraints and difficulties of the problem in $n$ dimensional space. We show a numerical example of the decomposition of a tensor of rank 3 with order one in each bunch of 3 variables in 3 dimensional space.

Contributions. We study the decomposition of multi-linear tensor $T$ as a sum of product of powers of linear forms in correlation with the decomposition of its dual $T^{*}$ as a weighted sum of evaluations. $T^{*}$ is defined via a formal power series $\tau$. We exploit the structure of the quotient algebra $A_{\tau}$ of the ring of multivariate polynomials in bunches of sub-variables by the kernel of the Hankel operator $H_{\tau}$ associated to $\tau$. We choose two bases $A_{1}$ and $A_{2}$ of monomials such that all given moments of the tensor $T$ appear in the matrix $H_{\tau}$ associated to $T$ in the bases $A_{1}$ and $A_{2}$ and we substitute $x_{0}$ by one. We compute the Singular Value Decomposition of the Hankel matrix associated to a chosen truncated bases of $A_{1}$ and $A_{2}$ such that the multiplication of the matrix by one fixed variable is well defined. We exploit the eigen-structure properties of multiplication operators to compute the sub-coordinates of points and their corresponding weights. We show the constraints which arise from the computation of all multiplication matrices in higher dimension spaces. We propose a new algorithm to compute the sub-coordinates of points using the eigenvalues of multiplication matrices and their transpose. We deduce weights from eigenvectors of a linear combination of multiplication matrices. This method is an adaptation of Structured Low Rank Decomposition of Multivariate Hankel Matrices method proposed in [7] which is the generalization of Prony method. We give a numerical interpretation of the decomposition of a multi-linear tensor of low rank in 3 dimensional space.

Structure of the paper. In the following section, we recall the definition of multi symmetric tensors of rank $r$ and the affine decomposition theory of them. In section 2, we recall some important properties of Artinian Gorenstein Algebra that we adapt to solve the dual decomposition problem which is resumed by the computation of points and their weights. In section 3 , we give a theoretical approach of the multi linear symmetric decomposition problem. In section 4, we propose a new algorithm to solve the decomposition problem in 3 dimensional space. Finally, we give an implementation of our algorithm for one example using MAPLE and we interpret the results.

\section{Partial Symmetric Tensor Decomposition Problem}

In this section we give the definition of a multi-symmetric tensor as a multi-homogeneous polynomial of a different positive degree at each collection of variables. This polynomial can be defined as well as multi symmetric array of coefficients. In the opposite, for a multi symmetric array of coefficients we can define a multi-homogeneous polynomial and then deshomogenize it. We recall the definition of minimal affine decomposition of a multi symmetric tensor as weighted sum of product of power of linear forms. We show the relationship between the dual of deshomogenized tensor and the formal power series associated to it using the apolar product. Then, after scaling by the linear form of the decomposition and multiplying the weights by the scaling factor we deduce by linearity that the dual of the Tensor can be decomposed as a weighted sum of evaluations.

Definition 1. Let $\left(E_{j}\right)_{1 \leq j \leq k}$ be a family of $n_{j}+1$ dimensional vector spaces, each one of them is of basis $\mathbf{x}_{j}$ such that $E_{j}=\left\langle\mathbf{x}_{j}\right\rangle=\left\langle x_{j}, \ldots, x_{j, n_{j}}\right\rangle$.

Definition 2. $\mathcal{S}^{\delta_{j}}\left(E_{j}\right)$ is the vector space of homogeneous polynomials in the variables $\mathbf{x}_{j}$ of degree $\delta_{j}$. 
Definition 3. $\mathcal{S}^{\delta_{1}}\left(E_{1}\right) \otimes \mathcal{S}^{\delta_{2}}\left(E_{2}\right) \otimes \ldots \otimes \mathcal{S}^{\delta_{k}}\left(E_{k}\right)$ is the vector space of multi-homogeneous polynomials of degree $\delta_{j}$ in each subset of variables $\mathbf{x}_{j}$ for $j=1, \ldots, k$, an element $[T]$ of this vector space is called a multi symmetric tensor. It is denoted hereafter as $\mathcal{S}^{\delta}(E)$.

Definition 4. A multi symmetric tensor of $\mathcal{S}^{\delta_{1}}\left(E_{1}\right) \otimes \mathcal{S}^{\delta_{2}}\left(E_{2}\right) \otimes \ldots \otimes \mathcal{S}^{\delta_{k}}\left(E_{k}\right)$ can be interpreted as a multi symmetric array of coefficients $[T]=\left[t_{\alpha_{1}^{\prime}, \alpha_{2}^{\prime}, \ldots, \alpha_{k}^{\prime}}\right]_{\left|\alpha_{j}^{\prime}\right|=\delta_{j}}$ such that each $\alpha_{j}^{\prime}=\left(\alpha_{j, p_{j}}^{\prime}\right)_{0 \leq p_{j} \leq n_{j}}$ is a multi-index for $1 \leq j \leq k$.$$
\alpha_{j}^{\prime} \in \mathbb{N}^{n_{j}+1}
$$

For $\alpha \in \mathbb{N}^{n}$ with $|\alpha| \leq \delta$, we denote $\bar{\alpha}=\left(\delta-|\alpha|, \alpha_{1}, \ldots, \alpha_{n}\right)$. The multi symmetric tensor is defined as $[T]=\left[t_{\bar{\alpha}_{1}, \bar{\alpha}_{1}, \ldots, \bar{\alpha}_{k}}\right]_{\substack{\alpha_{j} \mid \leq \delta_{j} \\ \alpha_{j} \in \mathbb{N}^{n_{j}}}}$.

Such tensor is identified with the multi-homogeneous polynomial

$$
T\left(\mathbf{x}_{1}, \mathbf{x}_{2}, \ldots, \mathbf{x}_{k}\right)=\sum_{\substack{\left|\bar{\alpha}_{j}\right|=\delta_{j} \\ \bar{\alpha}_{j} \in \mathbb{N}^{n} j^{+1}}} t_{\bar{\alpha}_{1}, \bar{\alpha}_{2}, \ldots, \bar{\alpha}_{k}}\left(\mathbf{x}_{1}\right)^{\bar{\alpha}_{1}}\left(\mathbf{x}_{2}\right)^{\bar{\alpha}_{2}} \ldots\left(\mathbf{x}_{k}\right)^{\bar{\alpha}_{k}}
$$

If we let $x_{j}=1$ for $j=1, \ldots, k$ we get

$$
\underline{T}\left(\underline{\mathbf{x}}_{1}, \underline{\mathbf{x}}_{2}, \ldots, \underline{\mathbf{x}}_{k}\right)=\sum_{\substack{\left|\alpha_{j}\right| \leq \delta_{j} \\ \alpha_{j} \in \mathbb{N}^{n_{j}}}} t_{\alpha_{1}, \alpha_{2}, \ldots, \alpha_{k}}\left(\underline{\mathbf{x}}_{1}\right)^{\alpha_{1}}\left(\underline{\mathbf{x}}_{2}\right)^{\alpha_{2}} \ldots\left(\underline{\mathbf{x}}_{k}\right)^{\alpha_{k}}
$$

where $\underline{\mathbf{x}}_{j}=\left(x_{j, 1}, \ldots, x_{j, n_{j}}\right)$ for $j=1, \ldots, k$ because of $\left(\underline{\mathbf{x}}_{j}\right)^{\alpha_{j}}=\left(\mathbf{x}_{j}\right)^{\bar{\alpha}_{j}}$ for $j=1, \ldots, k$.

A multilinear tensor is defined when $\left|\bar{\alpha}_{j}\right|=\delta_{j}=1$ for $j=1, \ldots, k$, then by abuse of notation we obtain $\bar{\alpha}_{j}\left[i_{j}\right]=1$ for some $0 \leq i_{j} \leq n_{j}$ and 0 elsewhere, so that the multi symmetric array associated to that tensor is defined as $[T]=\left[t_{i_{1}, i_{2}, \ldots, i_{k}}\right]_{0 \leq i_{j} \leq n_{j}}$

$1 \leq j \leq k$

Given $e_{j}$ basis of $E_{j}$ for $j=1, \ldots, k$, the tensor [T] in the basis $e_{1} \otimes e_{2} \otimes \ldots \otimes e_{k}$ is equal to $T=$ $\sum_{0 \leq i_{1} \leq n_{1}} t_{i_{1}, i_{2}, \ldots, i_{k}} e_{1, i_{1}} \otimes e_{2, i_{2}} \otimes \ldots \otimes e_{k, i_{k}}$, such a tensor can be identified with the multi-homogeneous poly$0 \leq i_{2} \leq n_{2}$

$0 \leq i_{k} \leq n_{k}$

nomial $T\left(\mathbf{x}_{1}, \mathbf{x}_{2}, \ldots, \mathbf{x}_{k}\right)=\sum_{0 \leq i_{1} \leq n_{1}} t_{i_{1}, i_{2}, \ldots, i_{k}} x_{1, i_{1}} x_{2, i_{2}} \ldots x_{k, i_{k}}$ because of $\left(\mathbf{x}_{j}\right)^{\bar{\alpha}_{j}}=x_{j, i_{j}}$ for some $0 \leq i_{j} \leq n_{j}$ $0 \leq i_{2} \leq n_{2}$

and for all $1 \leq j \leq k$.

$0 \leq i_{k} \leq n_{k}$

The dual of the tensor is $T^{*}\left(\mathbf{y}_{1}, \mathbf{y}_{2}, \ldots, \mathbf{y}_{k}\right)=\sum_{0 \leq i_{1} \leq n_{1}} t_{i_{1}, i_{2}, \ldots, i_{k}} y_{1, i_{1}} y_{2, i_{2}} \ldots y_{k, i_{k}}$ because of $\left(\mathbf{y}_{j}\right)^{\bar{\alpha}_{j}}=y_{j, i_{j}}$ $0 \leq i_{2} \leq n_{2}$

$0 \leq i_{k} \leq n_{k}$

for some $0 \leq i_{j} \leq n_{j}$ and for all $1 \leq j \leq k$.

We denote $R_{\delta_{1}, \delta_{2}, \ldots, \delta_{k}}$ the space obtained by the deshomogeneisation of elements in $\mathcal{S}^{\delta}(E)$ by setting $x_{j}=1$ for $j=1, \ldots, k$ where $R=\mathbb{C}\left[\underline{\mathbf{x}}_{1}, \underline{\mathbf{x}}_{2}, \ldots, \underline{\mathbf{x}}_{k}\right]$ is the space of polynomials in the variables $\underline{\mathbf{x}}_{j}=\left(x_{j, 1}, \ldots, x_{j, n_{j}}\right)$ for $j=1, \ldots, k$

Definition 5. The tensor decomposition problem of $T\left(\mathbf{x}_{1}, \mathbf{x}_{2}, \ldots, \mathbf{x}_{k}\right)$ is the decomposition of $T$ as a sum of product of power of linear forms such that $T\left(\mathbf{x}_{1}, \mathbf{x}_{2}, \ldots, \mathbf{x}_{k}\right)=\sum_{p=1}^{r} \omega_{p} \mathbf{u}_{p, 1}^{\delta_{1}}\left(\mathbf{x}_{1}\right) \mathbf{u}_{p, 2}^{\delta_{2}}\left(\mathbf{x}_{2}\right) \ldots \mathbf{u}_{i, k}^{\delta_{k}}\left(\mathbf{x}_{k}\right)$ where $\mathbf{u}_{p, j}\left(\mathbf{x}_{j}\right)=u_{p, j} x_{j}+u_{p, j, 1} x_{j, 1}+\ldots+u_{p, j, n_{j}} x_{j, n_{j}}$ and

$\mathbf{u}_{p}=\left(u_{p, j, p_{j}}\right)_{\substack{0 \leq p_{j} \leq n_{j} \\ 1 \leq j \leq k}}=\left(u_{p, 1}, u_{p, 1,1}, \ldots, u_{p, 1, n_{1}}, u_{p, 2}, u_{p, 2,1}, \ldots, u_{p, 2, n_{2}}, \ldots \ldots, u_{p, k}, u_{p, k, 1}, \ldots, u_{p, k, n_{k}}\right) \in \mathbb{C}^{\sum_{j=1}^{k}\left(n_{j}+1\right)}$ is the coefficient vector associated to the linear forms $\mathbf{u}_{p, j}\left(\mathbf{x}_{j}\right)$ in the basis $\mathbf{x}_{j}$ for $j=1, \ldots, k$.

Definition 6. The minimal number of terms in a decomposition of $T(\mathbf{x})$ is called the rank of $T$.

We say that $T\left(\mathbf{x}_{1}, \mathbf{x}_{2}, \ldots, \mathbf{x}_{k}\right)$ has an affine minimal decomposition of the previous form if $u_{p, j} \neq 0$ for $p=1, \ldots, r$ and $j=1, \ldots, k$ where $r$ is the rank of $T$. 
Definition 7. For $T=\left(t_{\alpha_{1}, \alpha_{2}, \ldots, \alpha_{k}}\right)_{\substack{\left|\alpha_{j}\right| \leq \delta_{j} \\ \alpha_{j} \in \mathbb{N}^{n_{j}}}} \in \mathcal{S}^{\delta}(E)$ we denote $\tau_{\alpha_{1}, \alpha_{2}, \ldots, \alpha_{k}}(T)=\tau_{\alpha_{1}, \alpha_{2}, \ldots, \alpha_{k}}=t_{\alpha_{1}, \alpha_{2}, \ldots, \alpha_{k}}\left(\begin{array}{c}\delta_{1} \\ \alpha_{1}\end{array}\right)^{-1}\left(\begin{array}{c}\delta_{2} \\ \alpha_{2}\end{array}\right)^{-1} \ldots\left(\begin{array}{c}\delta_{k} \\ \alpha_{k}\end{array}\right)^{-1}$. The dual of the tensor $T\left(\mathbf{x}_{1}, \mathbf{x}_{2}, \ldots, \mathbf{x}_{k}\right) \in \mathcal{S}^{\delta}(E)$ is defined via the formal power series as $\tau\left(\mathbf{y}_{1}, \mathbf{y}_{2}, \ldots, \mathbf{y}_{k}\right)=T^{*}\left(\mathbf{y}_{1}, \mathbf{y}_{2}, \ldots, \mathbf{y}_{k}\right)=\sum_{\substack{\left|\alpha_{j}\right| \leq \delta_{j} \\ \alpha_{j} \in \mathbb{N}^{n_{j}}}} \tau_{\alpha_{1}, \alpha_{2}, \ldots, \alpha_{k}} \frac{\left(\mathbf{y}_{1} \bar{\alpha}^{\bar{\alpha}_{1}} !\right.}{\bar{\alpha}_{1} !} \frac{\left(\mathbf{y}_{2}\right)^{\bar{\alpha}_{2}}}{\bar{\alpha}_{2} !} \ldots \frac{\left(\mathbf{y}_{k}\right)^{\bar{\alpha}_{k}}}{\bar{\alpha}_{k} !}$ where $\left(\mathbf{y}_{j}\right)^{\bar{\alpha}_{j}}=\left(y_{j}, y_{j, 1}, \ldots, y_{j, n_{j}}\right)^{\left(\alpha_{j}, \alpha_{j, 1}, \ldots, \alpha_{j, n_{j}}\right)}=\prod_{p_{j}=0}^{n_{j}}\left(y_{j, p_{j}}\right)^{\alpha_{j, p_{j}}}$ for $j=1, \ldots, k$

Definition 8. For a polynomial $p \in R$ and a formal power series $\tau \in R^{*}$, we define the multiplication operator * such that

$$
\begin{aligned}
p * \tau: R & \rightarrow \mathbb{C} \\
q & \mapsto \tau(p . q)
\end{aligned}
$$

Definition 9. Let $T_{1}\left(\mathbf{x}_{1}, \mathbf{x}_{2}, \ldots, \mathbf{x}_{k}\right)$ and $T_{2}\left(\mathbf{x}_{1}, \mathbf{x}_{2}, \ldots, \mathbf{x}_{k}\right)$ be two tensors of $\mathcal{S}^{\delta}(E)$. The apolar product of $\underline{T_{1}}\left(\underline{\mathbf{x}}_{1}, \underline{\mathbf{x}}_{2}, \ldots, \underline{\mathbf{x}}_{k}\right)$ and $\underline{T}_{2}\left(\underline{\mathbf{x}}_{1}, \underline{\mathbf{x}}_{2}, \ldots, \underline{\mathbf{x}}_{k}\right)$ is defined as

$$
\left\langle\underline{T_{1}}\left(\underline{\mathbf{x}}_{1}, \underline{\mathbf{x}}_{2}, \ldots, \underline{\mathbf{x}}_{k}\right), \underline{T_{2}}\left(\underline{\mathbf{x}}_{1}, \underline{\mathbf{x}}_{2}, \ldots, \underline{\mathbf{x}}_{k}\right)\right\rangle=\sum_{\substack{\left|\alpha_{j}\right| \leq \delta_{j} \\
\alpha_{j} \in \mathbb{N}^{n_{j}}}} \tau_{\alpha_{1}, \alpha_{2}, \ldots, \alpha_{k}}^{(1)} \bar{\tau}_{\alpha_{1}, \alpha_{2}, \ldots, \alpha_{k}}^{(2)}\left(\begin{array}{c}
\delta \\
\alpha
\end{array}\right) \text { where }\left(\begin{array}{c}
\delta \\
\alpha
\end{array}\right)=\left(\begin{array}{c}
\delta_{1} \\
\alpha_{1}
\end{array}\right)\left(\begin{array}{c}
\delta_{2} \\
\alpha_{2}
\end{array}\right) \ldots\left(\begin{array}{l}
\delta_{k} \\
\alpha^{k}
\end{array}\right) \text {. }
$$

Definition 10. The dual operator of a tensor is defined as

$$
\begin{aligned}
T^{*}:\left(R_{\delta_{1}, \delta_{2}, \ldots, \delta_{k}}\right) & \rightarrow\left(R_{\delta_{1}, \delta_{2}, \ldots, \delta_{k}}\right)^{*} \\
\underline{T_{2}} & \mapsto T^{*}\left(\underline{T_{2}}\right)=\left\langle\underline{T}(\underline{\mathbf{x}}), \underline{T_{2}}(\underline{\mathbf{x}})\right\rangle
\end{aligned}
$$

Lemma 1. By a generic change of coordinates in each $E_{j}$, we may assume that $u_{p, j} \neq 0$ and that $T$ has an affine decomposition. Then by scaling $\mathbf{u}_{p}(\mathbf{x})$ and multiplying $\omega_{p}$ by the $d^{\text {th }}$ power of the scaling factor we may assume that $u_{p, j}=1$ for $p=1, \ldots, r$ and $j=1, \ldots, k$. Thus the polynomial $\underline{T}(\underline{\mathbf{x}})=\sum_{p=1}^{r} \omega_{p}^{\prime} \mathbf{u}_{p}^{\prime} \delta(\underline{\mathbf{x}})=$ $\sum_{p=1}^{r} \omega_{i}^{\prime} \mathbf{u}_{p, 1}^{\prime} \delta_{1}\left(\underline{\mathbf{x}}_{1}\right) \mathbf{u}_{p, 2}^{\prime} \delta_{2}\left(\underline{\mathbf{x}}_{2}\right) \ldots \mathbf{u}_{p, k}^{\prime} \delta_{k}\left(\underline{\mathbf{x}}_{k}\right)$

Proposition 1. The dual of the product of powers of linear forms $\mathbf{u}_{1}^{\delta_{1}} \mathbf{u}_{2}^{\delta_{2}} \ldots \mathbf{u}_{k}^{\delta_{k}}$ is the evaluation $\mathbf{e}_{\mathbf{u}}$ at $\mathbf{u}=\left(\mathbf{u}_{1}, \mathbf{u}_{2}, \ldots, \mathbf{u}_{k}\right)$.

Proof. For $T=\mathbf{u}_{1}^{\delta_{1}} \mathbf{u}_{2}^{\delta_{2}} \ldots \mathbf{u}_{k}^{\delta_{k}}$ and any $T^{\prime} \in R_{\delta_{1}, \delta_{2}, \ldots, \delta_{k}}$, we check that $\left\langle\underline{T}(\underline{\mathbf{x}}), \underline{T^{\prime}}(\underline{\mathbf{x}})\right\rangle=T^{\prime}(\mathbf{u})$. This shows that $T^{*}$ coincides with the evaluation $\mathbf{e}_{\mathbf{u}}$.

Thus if $T=\sum_{i} \omega_{i} \mathbf{u}_{i, 1}^{\delta_{1}} \mathbf{u}_{i, 2}^{\delta_{2}} \ldots \mathbf{u}_{i, k}^{\delta_{k}}$, then $T^{*}$ coincides with the weighted sum of evalautions $T^{*}=\sum_{i} \omega_{i} \mathbf{e}_{b u_{i}}$ on $R_{\delta_{1}, \delta_{2}, \ldots, \delta_{k}}$. We reduce the decomposition problem of $T$ to the decomposition of $T^{*}$ as a weighted sum of evaluations $T^{*}=\sum_{i} \omega_{i} \mathbf{e}_{b u_{i}}$.

\subsection{Solving polynomial equations by eigenvector computation}

We recall the definition of quotient algebra $A$ of the ring of polynomials in a collection of variables $\mathbf{x}=$ $\left[\mathbf{x}_{1}, \mathbf{x}_{2}, \ldots, \mathbf{x}_{k}\right]$ where $\mathbf{x}_{j}=\left[\mathbf{x}_{j, 1}, \mathbf{x}_{j, 2}, \ldots, \mathbf{x}_{j, n_{j}}\right]$ for $j=1, \ldots, k$ by an ideal $I$ of multivariate polynomials. We use eigen-structure properties of multiplication operators and their transpose in a chosen basis of $A$ and its dual to compute the x's coordinates of points in the decomposition of the associated multi-linear tensor.

A quotient algebra $\mathcal{A}=\mathbb{C}[\mathbf{x}] / I$ is Artinian if it is of finite dimension over $\mathbb{C}$. In the case of partial symmetric tensor, the variables $\mathbf{x}$ and $\mathbf{y}$ are divided into bunches of sub-variables such that $\mathbf{x}=\left[\mathbf{x}_{1}, \mathbf{x}_{2}, \ldots, \mathbf{x}_{k}\right]$ and $\mathbf{y}=\left[\mathbf{y}_{1}, \mathbf{y}_{2}, \ldots, \mathbf{y}_{k}\right]$. Hereafter, in the case of multi-symmetric tensor the ideal $I$ defines a finite number of roots $\mathcal{V}(I)=\left\{\xi_{1}, \xi_{2}, \ldots, \xi_{r^{\prime}}\right\}=\left\{\xi \in \mathbb{C}^{n} \mid \forall q \in I, q(\xi)=0\right\}$ where $n=\sum_{j=1}^{k}\left(n_{j}+1\right)$ such that $n_{j}+1$ is the dimension of each vector space spanned by $\mathbf{x}_{j}$ and we have a decomposition of $\mathcal{A}$ as a sum of sub-algebras:

$$
\mathcal{A}=\mathbb{C}[\mathbf{x}] / I=\mathcal{A}_{1} \oplus \cdots \oplus \mathcal{A}_{r^{\prime}}
$$


where $\mathcal{A}_{p}=\mathbf{u}_{\xi_{p}} \mathcal{A} \sim \mathbb{C}[\mathbf{x}] / Q_{p}$ and $Q_{p}$ is the primary component of $I$ associated to the root $\xi_{p} \in \mathbb{C}^{n}$. The elements $\mathbf{u}_{1}, \ldots, \mathbf{u}_{r^{\prime}}$ satisfy the relations

$$
\mathbf{u}_{\xi_{p}}^{2}(\mathbf{x}) \equiv \mathbf{u}_{\xi_{p}}(\mathbf{x}), \sum_{i=1}^{r} \mathbf{u}_{\xi_{p}}(\mathbf{x}) \equiv 1
$$

The polynomials $\mathbf{u}_{\xi_{1}}, \ldots, \mathbf{u}_{\xi_{r^{\prime}}}$ are called idempotents of $\mathcal{A}$. The dimension of $\mathcal{A}_{p}$ is the multiplicity of the point $\xi_{p}$. For more details, see [6] [Chap. 4].

For $g \in \mathbb{C}[\mathbf{x}]$, the multiplication operator $\mathcal{M}_{g}$ is defined by

$$
\begin{aligned}
\mathcal{M}_{g}: \mathcal{A} & \rightarrow \mathcal{A} \\
h & \mapsto \mathcal{M}_{g}(h)=g h .
\end{aligned}
$$

The transpose $\mathcal{M}_{g}^{\top}$ of the multiplication operator $\mathcal{M}_{g}$ is

$$
\begin{aligned}
\mathcal{M}_{g}^{\top}: \mathcal{A}^{*} & \rightarrow \mathcal{A}^{*} \\
\Lambda & \mapsto \mathcal{M}_{g}^{\top}(\Lambda)=\Lambda \circ \mathcal{M}_{g}=g \star \Lambda .
\end{aligned}
$$

The main property that we will use to recover the roots is the following [6] [Thm. 4.23]:

Proposition 2. Let $I$ be an ideal of $\mathbb{C}[\mathbf{x}]$ and suppose that $\mathcal{V}(I)=\left\{\xi_{1}, \xi_{2}, \ldots, \xi_{r^{\prime}}\right\}$. Then

- for all $g \in \mathcal{A}$, the eigenvalues of $\mathcal{M}_{g}$ and $\mathcal{M}_{g}^{\top}$ are the values $g\left(\xi_{1}\right), \ldots, g\left(\xi_{r^{\prime}}\right)$ of the polynomial $g$ at the roots with multiplicities $\mu_{p}=\operatorname{dim} \mathcal{A}_{p}$.

- The eigenvectors common to all $\mathcal{M}_{g}^{\top}$ with $g \in \mathcal{A}$ are - up to a scalar - the evaluations $\mathbf{e}_{\xi_{1}}, \ldots, \mathbf{e}_{\xi_{r^{\prime}}}$.

If $B=\left\{b_{1}, \ldots, b_{r}\right\}$ is a basis of $\mathcal{A}$, then the coefficient vector of the evaluation $\mathbf{e}_{\xi_{p}}$ in the dual basis of $B$ is $\left[\left\langle\mathbf{e}_{\xi_{p}} \mid b_{j}\right\rangle\right]_{\beta \in B}=\left[b_{j}\left(\xi_{p}\right)\right]_{p=1 \ldots r}=B\left(\xi_{p}\right)$. The previous proposition says that if $M_{g}$ is the matrix of $\mathcal{M}_{g}$ in the basis $B$ of $\mathcal{A}$, then

$$
M_{g}^{\top} B\left(\xi_{p}\right)=g\left(\xi_{p}\right) B\left(\xi_{p}\right) .
$$

If moreover the basis $B$ contains the monomials $1, x_{1,1}, x_{1,2}, \ldots, x_{1, n_{1}}$, then the common eigenvectors of $M_{g}^{\top}$ are of the form $\mathbf{v}_{p}=c\left[1, \xi_{p, 1,1}, \ldots, \xi_{p, 1, n_{1}}, \ldots\right]$ and the x's coordinates of the root $\xi_{p}$ can be computed from the coefficients of $\mathbf{v}_{p}$ by taking the ratio of the coefficients of the monomials $x_{1,1}, \ldots, x_{1, n_{1}}$ by the coefficient of $1: \xi_{p, 1, i_{1}}=\frac{\mathbf{v}_{p, 1, i_{1}+1}}{\mathbf{v}_{p, 1,1}}$. Thus computing the common eigenvectors of all the matrices $M_{g}^{\top}$ for $g \in \mathcal{A}$ yield the x's coordinates of the roots $\xi_{p}(p=1, \ldots, r)$.

In practice, it is enough to compute the common eigenvectors of $M_{x_{1,1}}^{\top}, \ldots, M_{x_{1, n_{1}}}^{\top}$, since $\forall g \in \mathbb{C}\left[\mathbf{x}_{1}\right], M_{g}^{\top}=$ $g\left(M_{x_{1,1}}^{\top}, \ldots, M_{x_{1, n_{1}}}^{\top}\right)$. Therefore, the common eigenvectors $M_{x_{1,1}}^{\top}, \ldots, M_{x_{1, n_{1}}}^{\top}$ are also eigenvectors of any $M_{g}^{\top}$.

The multiplicity structure, that is the dual $Q_{p}^{\perp}$ of each primary component $Q_{p}$ of $I$, also called the inverse system of the point $\xi_{p}$ can be deduced by linear algebra tools (see e.g. [13]).

In the case of simple roots, we have the following property [6] [Chap. 4]:

Proposition 3. If the roots $\left\{\xi_{1}, \xi_{2}, \ldots, \xi_{r}\right\}$ of I are simple (i.e. $\mu_{p}=\operatorname{dim} \mathcal{A}_{p}=1$ ) then we have the following:

$-\mathbb{u}=\left\{\mathbf{u}_{\xi_{1}}, \ldots, \mathbf{u}_{\xi_{r}}\right\}$ is a basis of $\mathcal{A}$.

- The polynomials $\mathbf{u}_{\xi_{1}}, \ldots, \mathbf{u}_{\xi_{r}}$ are interpolation polynomials at the roots $\xi_{p}$ : $\mathbf{u}_{\xi_{p}}\left(\xi_{q}\right)=1$ if $p=q$ and 0 otherwise.

- The matrix of $\mathcal{M}_{g}$ in the basis $\mathbb{u}$ is the diagonal matrix $\operatorname{diag}\left(g\left(\xi_{1}\right), \ldots, g\left(\xi_{r}\right)\right)$.

This proposition tells us that if $g$ is separating the roots, i.e. $g\left(\xi_{p}\right) \neq g\left(\xi_{q}\right)$ for $p \neq q$, then the eigenvectors of $\mathcal{M}_{g}$ are, up to a scalar, interpolation polynomials at the roots. 


\subsection{Artinian Gorenstein algebra of a multivariate Hankel operator}

In this section, we detail the construction of the quotient algebra $A_{\tau}$ by the kernel $I_{\tau}$ of the Hankel operator $H_{\tau}$ associated to the dual of the tensor $T$. We compute a basis of $A_{\tau}$ such that the submatrix associated to it has a maximal non-zero minor of a truncated matrix of $H_{\tau}$. We recall how to compute the multiplication matrices in this associated basis and its dual using some shifted submatrices of $H_{\tau}$. We notice that not all of them are easy to compute. We benefit from properties of generalized eigenvalues of multiplication matrices by $y^{\prime} s$ to compute the x's coordinates of points. We show how to use the generalized eigenvectors of the multiplication matrices to compute the weights.

We associate to a Hankel operator $H_{\tau}$, the quotient $\mathcal{A}_{\tau}=\mathbb{C}[\mathbf{x}] / I_{\tau}$ of the polynomial ring $\mathbb{C}[\mathbf{x}]$ modulo the kernel $I_{\tau}=\{p \in \mathbb{C}[\mathbf{x}] \mid \forall q \in R,\langle\tau \mid p q\rangle=0\}$ of $H_{\tau}$. We check that $I_{\tau}$ is an ideal of $\mathbb{C}[\mathbf{x}]$, so that $\mathcal{A}_{\tau}$ is an algebra.

As $\mathcal{A}_{\tau}=\mathbb{C}[\mathbf{x}] / I_{\tau} \sim \operatorname{img} H_{\tau}$, the operator $H_{\tau}$ is of finite rank $r$, if and only if, $\mathcal{A}_{\tau}$ is Artinian of dimension $\operatorname{dim}_{\mathbb{C}} \mathcal{A}_{\tau}=r$.

A quotient algebra $\mathcal{A}$ is called Gorenstein if its dual $\mathcal{A}^{*}=\operatorname{Hom}_{\mathbb{C}}(\mathcal{A}, \mathbb{C})$ is a free $\mathcal{A}$-module generated by one element.

In our context, we have the following equivalent properties [14]:

- $\tau=\sum_{p=1}^{r^{\prime}} \omega_{p}(\mathbf{y}) \mathbf{e}_{\xi_{p}}(\mathbf{y})$ with $\omega_{p} \in \mathbb{C}[\mathbf{y}], \xi_{p} \in \mathbb{C}^{n}$ and $\sum_{p=1}^{r^{\prime}} \mu\left(\omega_{p}\right)=r$ where $n=\sum_{j=1}^{k}\left(n_{j}+1\right)$,

- $H_{\tau}$ is of rank $r$,

- $\mathcal{A}_{\tau}$ is an Artinian Gorenstein algebra of dimension $r$.

Another property that will be helpful to determine a basis of $\mathcal{A}_{\tau}$ is the following:

Lemma 2. Let $B=\left\{b_{1}, \ldots, b_{r}\right\}, B^{\prime}=\left\{b_{1}^{\prime}, \ldots, b_{r}^{\prime}\right\} \subset \mathbb{C}[\mathbf{x}]$. If the matrix $H_{\tau}^{B, B^{\prime}}=\left(\left\langle\tau \mid b_{p} b_{q}^{\prime}\right\rangle\right)_{1 \leq p, q \leq r}$ is invertible, then $B$ and $B^{\prime}$ are linearly independent in $\mathcal{A}_{\tau}$.

By this Lemma, bases of $\mathcal{A}_{\tau}$ can be computed by identifying non-zero minors of maximal size of the matrix of $H_{\tau}$.

Proposition 4. Let $B, B^{\prime}$ be basis of $\mathcal{A}_{\tau}$ and $g \in \mathbb{C}[\mathbf{x}]$. We have

$$
H_{g \star \tau}^{B, B^{\prime}}=\left(M_{g}^{B}\right)^{\top} H_{\tau}^{B, B^{\prime}}=H_{\tau}^{B, B^{\prime}} M_{g}^{B^{\prime}} .
$$

where $M_{g}^{B}$ (resp. $M_{g}^{B^{\prime}}$ ) is the matrix of the multiplication by $g$ in the basis $B$ (resp. $B^{\prime}$ ) of $\mathcal{A}_{\tau}$.

We deduce the following property:

Proposition 5. Let $\tau(\mathbf{y})=\sum_{p=1}^{r} \omega_{p}(\mathbf{y}) \mathbf{e}_{\xi_{p}}(\mathbf{y})$ with $\omega_{p} \in \mathbb{C}[\mathbf{y}] \backslash\{0\}$ and $\xi_{p} \in \mathbb{C}^{n}$ distinct and let $B, B^{\prime}$ be bases of $\mathcal{A}_{\tau}$. We have the following properties:

- For $g \in \mathbb{C}[\mathbf{x}], M_{g}^{B^{\prime}}=\left(H_{\tau}^{B, B^{\prime}}\right)^{-1} H_{g \star \tau}^{B, B^{\prime}},\left(M_{g}^{B}\right)^{\top}=H_{g \star \tau}^{B, B^{\prime}}\left(H_{\tau}^{B, B^{\prime}}\right)^{-1}$.

- For $g \in \mathbb{C}[\mathbf{x}]$, the generalized eigenvalues of $\left(H_{g \star \tau}^{B, B^{\prime}}, H_{\tau}^{B, B^{\prime}}\right)$ are $g\left(\xi_{p}\right)$ with multiplicity $\mu_{p}=\mu\left(\omega_{p}\right)$, $p=1, \ldots, r$.

- The generalized eigenvectors common to all $\left(H_{g \star \tau}^{B, B^{\prime}}, H_{\tau}^{B, B^{\prime}}\right)$ for $g \in \mathbb{C}[\mathbf{x}]$ are - up to a scalar $-\left(H_{\tau}^{B, B^{\prime}}\right)^{-1} B\left(\xi_{p}\right)$, $p=1, \ldots, r$.

Proof. The two first points are direct consequences of Propositions 4 and 2 . The third point is also a consequence of Proposition 2, since the coordinate vector of the evaluation $\mathbf{e}_{\xi_{p}}$ in the dual basis of $B$ is $B\left(\xi_{p}\right)$ for $p=1, \ldots, r$.

This proposition shows that the matrices of multiplication by an element $g$ in $\mathcal{A}$, and thus the roots $\left\{\xi_{1}, \ldots, \xi_{r}\right\}$ and their multiplicity structure, can be computed from truncated Hankel matrices, provided we can determine bases $B, B^{\prime}$ of $\mathcal{A}_{\tau}$. In practice, it is enough to compute the generalized eigenvectors common to $\left(H_{x_{1, i_{1} \star \tau}}^{B, B^{\prime}}, H_{\tau}^{B, B^{\prime}}\right)$ for $i_{1}=1, \ldots, n_{1}$ to recover the roots. As $H_{x_{1, i_{1}} \star \tau}^{B, B^{\prime}}=H_{\tau}^{x_{1, i_{1}} B, B^{\prime}}=H_{\tau}^{B, x_{1, i_{1}} B^{\prime}}$, the decomposition can be computed from sub-matrices of $H_{\tau}^{B, B^{\prime+}}$ or $H_{\tau}^{B^{+}, B^{\prime}}$ where $B^{+}=B \cup x_{1,1} B \cup \cdots \cup x_{1, n_{1}} B$, $B^{\prime+}=B^{\prime} \cup x_{1,1} B^{\prime} \cup \cdots \cup x_{1, n_{1}} B^{\prime}$. 


\section{Multilinear Tensor Decomposition Problem}

In this section, we analyze the easiest case of multi symmetric tensor where it is of degree one at each bunch of sub-variables. Our goal is to decompose $\tau$ which is equal to $T^{*}$ as a weigthed sum of evaluations by computing the eigen-structure of $\mathcal{A}_{\tau}$ which is based on the computation of multiplication operators. We simplify notations by using subscripts of variables and coefficients instead of multi-index exponents. We compute the truncated Singular Value Decomposition of a generic linear combination of a shifted Hankel matrices by the first collection of variables. By linearity and properties of the multiplication operators by one variable described in section 2 , we deduce the multiplication operators by more complex variables which could be used to compute weights and points.

We choose two monomial bases $B_{1}$ and $B_{2}$ indexing respectively rows and columns of the Hankel matrix $H_{T^{*}}^{B_{1}, B_{2}}$ associated to the tensor $T^{*}$, such that the set of monomials $\left\{B_{1} * B_{2} x_{j, i_{j}}, 0 \leq i_{j} \leq n_{j}, 1 \leq j \leq k\right\}$ span the set of deshomogenized polynomials $R_{\delta_{1}, \delta_{2}, \ldots, \delta_{k}}$.

The matrix of the truncated Hankel operator in the basis $B_{1}$ and the dual basis $B_{2}$ is

$$
H_{T^{*}}^{B_{1}, B_{2}}=\left[t_{i_{1}, i_{2}, \ldots, i_{k}}\right]_{\substack{0 \leq i_{1} \leq n_{1} \\ 0 \leq i_{2} \leq n_{2} \\ i \\ 0 \leq i_{k} \leq n_{k}}} .
$$

The Hankel matrix associated to the tensor $x_{1, i_{1}} * T^{*}$ is defined as $H_{1, i_{1}}=H_{x_{1, i_{1}} * T^{*}}^{B_{1}, B_{2}}=H_{T^{*}}^{x_{1, i_{1}} * B_{1}, B_{2}}=$ $\left[t_{\alpha+\beta}\right]_{\alpha \in x_{1, i_{1}} * B_{1}, \beta \in B_{2}}$, all the elements of the matrix are divisible in $x_{1, i_{1}}$ and of degree $\delta$.

For example, the Hankel matrix associated to $x_{1} * T^{*}$ in the monomials basis $B_{1}$ and $B_{2}$ is denoted by $H_{0}$. Let $\lambda\left(\mathbf{x}_{1}\right)=\lambda_{0}+\lambda_{1} x_{1,1}+\ldots+\lambda_{n_{1}} x_{1, n_{1}}$ is a linear form with generic chosen coefficients $\lambda_{i_{1}}, i_{1}=0, \ldots, n_{1}$, we build a linear combination of $H_{1, i_{1}}, i_{1}=0, \ldots, n_{1}$ such that $\widehat{H}_{0}=\sum_{i_{1}=0}^{n_{1}} \lambda_{i_{1}} H_{1, i_{1}}$ we compute the singular value decomposition of it.

Computing the singular value decomposition of $\widehat{H}_{0}$, we obtain

$$
\widehat{H}_{0}=U S V^{\top}
$$

where $S$ is the diagonal matrix of all singular values of $\widehat{H}_{0}$ arranged in a decreasing order, $U$ is an unitary matrix whose columns are the left singular vectors of $\widehat{H}_{0}, V$ is an unitary matrix whose columns are the right singular vectors of $\widehat{H}_{0}$. We denote by $U^{\mathrm{H}}$ the hermitian transpose of $U$ and $\bar{V}$ the conjugate of $V$. We denote by $U_{r}$ and $V_{r}$ the truncated matrices of the first $r$ columns of $U$ and $V$ and $S_{r}$ the diagonal matrix of the first $r$ rows and $r$ columns of $S$.

We denote $\bar{B}_{1}=\left\langle 1, x_{1,1}, \ldots, x_{1, n_{1}}\right\rangle$ and $\bar{B}_{2}=\left\langle 1, x_{k, 1}, \ldots, x_{k, n_{k}}\right\rangle$. Let $u_{i}=\left[u_{\alpha, i}\right]_{\alpha \in \bar{B}_{1}}$ and $v_{j}=\left[v_{\beta, j}\right]_{\beta \in \bar{B}_{2}}$ be respectively the $i^{\text {th }}$ and $j^{\text {th }}$ columns of $U^{\mathrm{H}}$ and $\bar{V}$. We denote by $u_{i}\left(\mathbf{x}_{1}\right)=u_{i}^{\top} U_{r}^{\mathrm{H}}$ and $v_{j}\left(\mathbf{x}_{1}\right)=v_{j}^{\top} \bar{V}_{r}$ the corresponding polynomials. The bases formed by these first $r$ polynomials are denoted $U_{r}^{\mathrm{H}}:=\left(u_{i}\left(\mathbf{x}_{1}\right)\right)_{i=1, \ldots, r}$ and $\bar{V}_{r}:=\left(v_{j}\left(\mathbf{x}_{1}\right)\right)_{j=1, \ldots, r}$. We will also denote by $U_{r}^{\mathrm{H}}\left(\right.$ resp. $\left.\bar{V}_{r}\right)$ the corresponding coefficient matrix, formed by the first rows (resp. columns) of $U^{\mathrm{H}}$ (resp. $\bar{V}$ ). We denote by $S_{r}$ the diagonal matrix of the first $r$ rows and columns of $S$, formed by the first $r$ singular values.

We denote by $H_{0}^{r}, H_{1, i_{1}}^{r}$ and $\widehat{H}_{0}^{r}$ the matrices obtained by the truncated singular value decomposition of $H_{0}, H_{i_{1}}$ and $\widehat{H}_{0}$ respectively.

We have the following property

$$
H_{i_{1}}^{r}=\left(M_{x_{1, i_{1}}}^{U_{r}^{\mathrm{H}}}\right)^{\top} H_{0}^{r}=H_{0}^{r} M_{x_{1, i_{1}} * T}^{\bar{V}_{r}}
$$

where $M_{x_{1, i_{1}}}^{U_{r}^{\mathrm{H}}}$ (resp. $M_{x_{1, i_{1}}}^{\bar{V}_{r}}$ ) is the multiplication matrix by $x_{1, i_{1}}$ in the basis $U_{r}^{\mathrm{H}}$ (resp. $\bar{V}_{r}$ ) and $M_{x_{1, i_{1} * T} \bar{V}_{r}}$ is the multiplication matrix by $x_{1, i_{1}} * T$ in the basis $\bar{V}_{r}$. Then by linearity, we obtain $\widehat{H}_{0}^{r}=\sum_{i_{1}=0}^{n_{1}} \lambda_{i_{1}} H_{1, i_{1}}^{r}=$ $H_{0}^{r} \sum_{i_{1}=0}^{n_{1}} \lambda_{i_{1}} M_{x_{1, i_{1} * T} \bar{V}_{r}}=H_{0}^{r} M_{\lambda\left(\mathbf{x}_{1}\right) * T}^{\bar{V}_{r}}$.

Then $\left(\widehat{H}_{0}^{r}\right)^{-1}=\left(M_{\lambda\left(\mathbf{x}_{1}\right) * T}^{\bar{V}_{r}}\right)^{-1}\left(H_{0}^{r}\right)^{-1}$ so multiplying by the first equation we get

$$
\left(\widehat{H}_{0}^{r}\right)^{-1} H_{1, i_{1}}^{r}=\left(M_{\lambda\left(\mathbf{x}_{1}\right) * T}^{\bar{V}_{r}}\right)^{-1} M_{x_{1, i_{1}} * T}^{\bar{V}_{r}}=M_{\left(x_{1, i_{1}} / \lambda\left(\mathbf{x}_{1}\right)\right) * T}^{\bar{V}_{r}}
$$


We compute the eigenvalues and the eigenvectors of the multiplication matrices $M_{\left(x_{1, i_{1}} / \lambda\left(\mathbf{x}_{1}\right)\right) * T}^{\bar{V}_{r}}$ in order to obtain the weights and the points of the decomposition.

\subsection{Algorithm}

We describe now the algorithm to recover the sum $\underline{T}^{*}(\underline{\mathbf{x}}, \underline{\mathbf{y}}, \underline{\mathbf{z}})=\sum_{p=1}^{r} \omega_{p} \mathbf{e}_{\mathbf{u}_{p}}(\underline{\mathbf{x}}, \underline{\mathbf{y}}, \underline{\mathbf{z}}), \omega_{p} \in \mathbb{C} \backslash\{0\}, \mathbf{u}_{p} \in$ $\mathbb{C}^{\sum_{l=1}^{3}\left(n_{l}+1\right)}$, from the moments of degree at most one at each bunch of coordinates $\left(t_{i, j, k}\right)_{\substack{0 \leq i \leq n_{1} \\ 0 \leq j \leq n_{2} \\ 0 \leq k \leq n_{3}}}$ of the formal

power series. To simplify, we change notations to better understand the nine dimensional multivariate space seen as three dimensional space. We only use 3 bunches of variables such that $x_{1, i_{1}}$ by $x_{i}$ and $x_{2, i_{2}}$ by $y_{j}$ and $x_{3, i_{3}}$ by $z_{k}$.

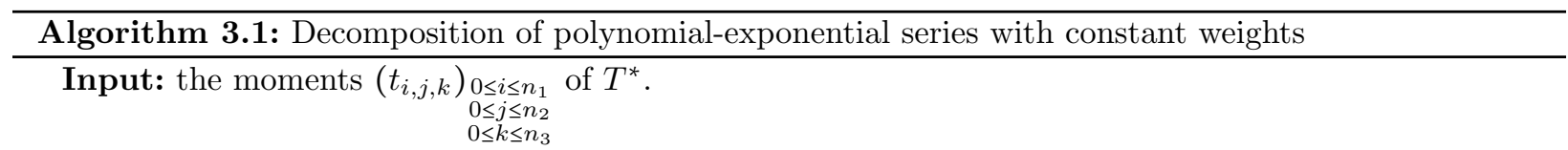

1. Compute the monomial sets $A_{1}=\left(x_{i} y_{j}\right)_{\substack{0 \leq i \leq n_{1} \\ 0 \leq j \leq n_{2}}}$ and $A_{2}=\left(z_{0}, z_{1}, \ldots, z_{n_{3}}\right)$ and substitute the $x_{0}, y_{0}$ and $z_{0}$ by 1 to define $B_{1}$ and $B_{2}$.

2. Compute the Hankel matrix $H_{T^{*}}^{B_{1}, B_{2}}=\left[t_{i, j, k}\right]_{\substack{0 \leq i \leq n_{1} \\ 0 \leq j \leq n_{2}}}$ for the monomial sets $B_{1}$ and $B_{2}$. $0 \leq j \leq n_{2}$

3. Compute the singular value decomposition of $H_{T^{*}}^{\bar{B}_{1}, \bar{B}_{2}}=U S V^{\top}$ where $\bar{B}_{1}=\left\langle 1, x_{1}, \ldots, x_{n_{1}}\right\rangle$ and $\bar{B}_{2}=\left\langle 1, z_{1}, \ldots, z_{n_{3}}\right\rangle$ with singular values $s_{1} \geq s_{2} \geq \cdots \geq s_{m} \geq 0$.

4. Determine its numerical rank, that is, the largest integer $r$ such that $\frac{s_{r}}{s_{1}} \geq \epsilon$.

5. Form the multiplication matrices by $y_{j}$ in the basis $\bar{V}_{r}, M_{y_{j}}^{\bar{V}_{r}}=S_{r}^{-1} U_{r}^{\mathrm{H}} H_{y_{j} * T^{*}}^{\bar{B}_{1}} \bar{V}_{r} \bar{V}_{r}$ where $H_{y_{j} * T^{*}}^{\bar{B}_{1}, \bar{B}_{2}}$ is the Hankel matrix associated to $y_{j} \star T^{*}$ for $j=1, \ldots, n_{2}$.

6. Compute the eigenvectors $\mathbf{v}_{p}$ of $\sum_{j=1}^{n_{2}} l_{j} M_{y_{j}}^{\bar{V}_{r}}$ such that $\left|l_{j}\right| \leq 1, j=1, \ldots, n_{2}$ and for each $p=1, \ldots, r$ do the following:

- The $y^{\prime} s$ coordinates of the $\mathbf{u}_{p}$ are the eigenvalues of the multiplication matrices by $y_{j}$. Use the formula $M_{y_{j}}^{\bar{V}_{r}} \mathbf{v}_{p}=u_{p, 2, j} \mathbf{v}_{p}$ for $p=1, \ldots, r$ and $j=1, \ldots, n_{2}$ and deduce the $u_{p, 2, j}$.

- Write the matrix $H_{T^{*}}^{\bar{B}_{1}, \bar{B}_{2}}$ in the basis of interpolation polynomials(ie. the eigenvectors $\mathbf{v}_{p}$ ) and use the corresponding matrix $\mathcal{T}$ to compute the $z^{\prime} s$ coordinates. Divide the $k^{\text {th }}$ row on the first row of the matrix $\mathcal{T}$ to obtain the values of $u_{p, 3, k}$ for $p=1, \ldots, r$ and $k=1, \ldots, n_{3}$.

- The $x^{\prime} s$ coordinates of $\mathbf{u}_{p}$ are computed using the eigenvectors of the transpose of the matrix $M_{y_{j}}^{\bar{V}_{r}}$. They -are up to scalar- the evaluations, they are represented by vectors of the form $\mathbf{v}_{p}^{*}=\mu_{p}\left[1, u_{p, 1,1}, \ldots, u_{p, 1, n_{1}}\right]$. Compute $\mathbf{v}_{p}^{*}$ as the $p^{t h}$ column of the transpose of the inverse of the matrix $V=\left[v_{1}, \ldots, v_{r}\right]$ for $p=1, \ldots, r$ and deduce $u_{p, 1, i}=\frac{\mathbf{v}_{p}^{*}[i+1]}{\mathbf{v}_{p}^{*}[1]}$ for $p=1, \ldots, r$ and $i=1, \ldots, n_{1}$.

- Compute $\omega_{p}=\frac{\left\langle T^{*} \mid \mathbf{v}_{p}\right\rangle}{\mathbf{v}_{p}\left(\mathbf{u}_{p}\right)}$.

Output: $r \in \mathbb{N}, \omega_{p} \in \mathbb{C} \backslash(0), \mathbf{u}_{p} \in \mathbb{C}^{\sum_{l=1}^{3}\left(n_{l}+1\right)}, p=1, \ldots, r$ such that $\underline{T}^{*}(\underline{\mathbf{x}}, \underline{\mathbf{y}}, \underline{\mathbf{z}})=\sum_{p=1}^{r} \omega_{p} \mathbf{e}_{\mathbf{u}_{p}}(\underline{\mathbf{x}}, \underline{\mathbf{y}}, \underline{\mathbf{z}})$ up to degree one at each bunch of coordinates.

The cost of the SVD computation is in $\mathcal{O}\left(s^{3}\right)$ where $s \geq r$ is the maximal size of the Hankel matrix $H_{0}$ and $r$ the rank of the decomposition. The computation of each multiplication matrice is in $\mathcal{O}\left(r^{2}\right)$ and the eigencomputation is in $\mathcal{O}\left(r^{3}\right)$. This yields a complexity bound in $\mathcal{O}\left(s^{3}+n r^{2}\right)$ for the complete algorithm, where $n=\max \left(n_{1}, n_{2}, n_{3}\right)$ is a bound on the dimension of the spaces. This complexity bound extends to the decomposition of general multi-symmetric tensors, provided $r=\operatorname{rank} H_{0}$. 


\section{Example}

In this section, we illustrate the decomposition algorithm on a multi-linear tensor of degree one at each bunch of 3 variables and of rank 3 .

If $\delta_{l}=1$ for all $l=1, \ldots, k, k>1$ and $n_{l}=n$, let $k=3, n_{l}=n=2, r=3$ and $\delta_{l}=1$ then we have $\mathbf{x}=\left(x_{0}, x_{1}, x_{2}\right), \mathbf{y}=\left(y_{0}, y_{1}, y_{2}\right)$ and $\mathbf{z}=\left(z_{0}, z_{1}, z_{2}\right)$. For

$\bar{\alpha} \in \mathbb{N}^{3},|\bar{\alpha}|=1 \Rightarrow \bar{\alpha}=(1),(0,1),(0,1) \Rightarrow \mathbf{x}^{\bar{\alpha}}=x_{i}, i=0, \ldots, 2$
$\bar{\beta} \in \mathbb{N}^{3},|\bar{\beta}|=1 \Rightarrow \bar{\beta}=(1),(0,1),(0,1) \Rightarrow \mathbf{y}^{\bar{\beta}}=y_{j}, j=0, \ldots, 2$
$\bar{\gamma} \in \mathbb{N}^{3},|\bar{\gamma}|=1 \Rightarrow \bar{\gamma}=(1),(0,1),(0,1) \Rightarrow \mathbf{z}^{\bar{\gamma}}=z_{k}, k=0, \ldots, 2$

The multi symmetric tensor is defined by a multi symmetric array of coefficients such that $t_{\alpha, \beta, \gamma}:=t_{\bar{\alpha}, \bar{\beta}, \bar{\gamma}}=$

$t_{i, j, k}$ then $T(\mathbf{x}, \mathbf{y}, \mathbf{z})=\sum_{\substack{0 \leq i \leq 2 \\ 0 \leq 5 \leq 2 \\ 0 \leq k \leq 2}} t_{i, j, k} x_{i} y_{j} z_{k}=0.4461757334 x_{0} y_{0} z_{0}-0.2262004083 x_{0} y_{0} z_{1}+0.4427031740 x_{0} y_{0} z_{2}-$

$0.2756785277 x_{0} y_{1} z_{0}+0.1612318550 x_{0} y_{1} z_{1}-0.3100164212 x_{0} y_{1} z_{2}-0.1209490221 x_{0} y_{2} z_{0}+0.1465160338 x_{0} y_{2} z_{1}-$

$0.1169341103 x_{0} y_{2} z_{2}-0.01239930649 x_{1} y_{0} z_{0}-0.05189330981 x_{1} y_{0} z_{1}+0.01803564422 x_{1} y_{0} z_{2}-0.01336683543 x_{1} y_{1} z_{0}+$

$0.02632784503 x_{1} y_{1} z_{1}-0.02598209626 x_{1} y_{1} z_{2}-0.3195263612 x_{1} y_{2} z_{0}+0.09311605022 x_{1} y_{2} z_{1}-0.1116610246 x_{1} y_{2} z_{2}-$

$0.1460187051 x_{2} y_{0} z_{0}+0.06557223848 x_{2} y_{0} z_{1}-0.1734312692 x_{2} y_{0} z_{2}+0.1010145926 x_{2} y_{1} z_{0}-0.05743078561 x_{2} y_{1} z_{1}+$

$0.1238292801 x_{2} y_{1} z_{2}-0.1485221955 x_{2} y_{2} z_{0}+0.03231415762 x_{2} y_{2} z_{1}-0.376925099 e-2 x_{2} y_{2} z_{2}$.

Let $x_{0}=y_{0}=z_{0}=1$ then $\underline{T}(\underline{\mathbf{x}}, \underline{\mathbf{y}}, \underline{\mathbf{z}})=\sum_{\substack{1 \leq i \leq 2 \\ 1 \leq j \leq 2 \\ 1 \leq k \leq 2}} t_{i, j, k} \underline{x}_{i} \underline{y}_{j} \underline{z}_{k}$

Then the tensor decomposition problem is $\underline{T}(\underline{\mathbf{x}}, \underline{\mathbf{y}}, \underline{\mathbf{z}})=\sum_{p=1}^{r} \omega_{p} \mathbf{u}_{p, 1}(\underline{\mathbf{x}}) \mathbf{u}_{p, 2}(\underline{\mathbf{y}}) \mathbf{u}_{p, 3}(\underline{\mathbf{z}})$. Given all the moments of degree at most one at each bunch of coordinates $\left(t_{i, j, k}\right)_{\substack{0 \leq i \leq 2 \\ 0 \leq j \leq 2}}$,

$$
\begin{aligned}
& 0 \leq j \leq 2 \\
& 0 \leq k \leq 2
\end{aligned}
$$

We create two sets $A_{1}=\left(\mathbf{x}^{\bar{\alpha}} \mathbf{y}^{\bar{\beta}}\right)_{\substack{|\bar{\alpha}|=1 \\|\bar{\beta}|=1}}=\left(x_{i} y_{j}\right)_{\substack{0 \leq i \leq 2 \\ 0 \leq j \leq 2}}$ and $A_{2}=\left(\mathbf{z}^{\bar{\gamma}}\right)_{|\bar{\gamma}|=1}=\left(z_{k}\right)_{0 \leq k \leq 2}$ so that

$A_{1}=\left(x_{0} y_{0}, x_{0} y_{1}, x_{0} y_{2}, x_{1} y_{0}, x_{1} y_{1}, x_{1} y_{2}, x_{2} y_{0}, x_{2} y_{1}, x_{2} y_{2}\right)$ and $A_{2}=\left(z_{0}, z_{1}, z_{2}\right)$.

For $x_{0}=y_{0}=z_{0}=1$ then $B_{1}=\left(1, y_{1}, y_{2}, x_{1}, x_{1} y_{1}, x_{2}, x_{2} y_{1}, x_{2} y_{2}\right)$ and $B_{2}=\left(1, z_{1}, z_{2}\right)$, the Hankel matrix associated to the tensor in the monomial basis $B_{1}$ and $B_{2}$ is

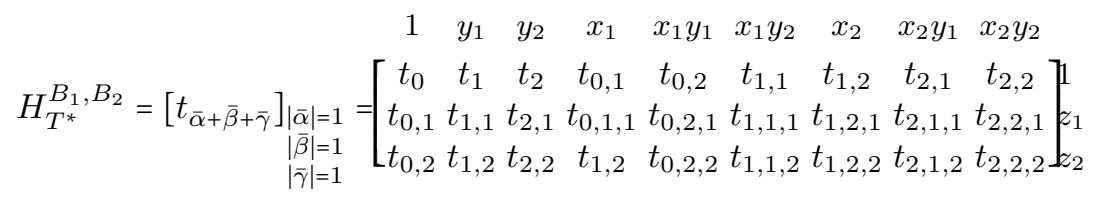

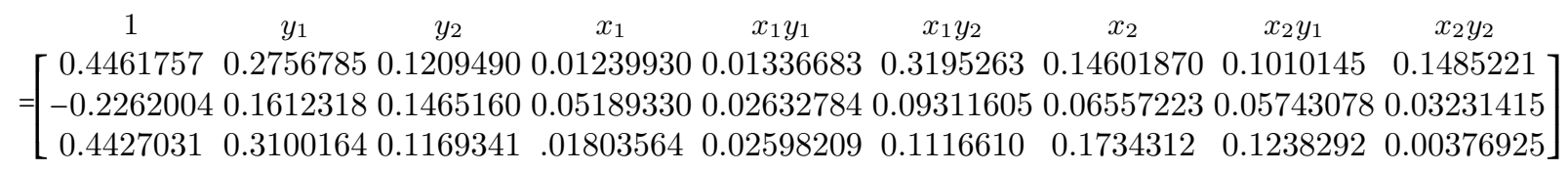

All the entries of this matrix are known, we choose $\bar{B}_{1}=\left\langle 1, x_{1}, x_{2}\right\rangle$ and $\bar{B}_{2}=\left\langle 1, z_{1}, z_{2}\right\rangle$ to be able to multiply by $y_{1}$ and to compute the multiplication matrix. Computing the singular value decomposition of $H_{T^{*}}^{\bar{B}_{1}, \bar{B}_{2}}$, we obtain

$$
\begin{aligned}
& \begin{array}{rrrrrrr}
1 & x_{1} & x_{2} & 1 & x_{1} & x_{2}
\end{array} \\
& H_{T^{*}}^{\bar{B}_{1}, \bar{B}_{2}}=U S V^{\top}=\left[\begin{array}{ccc}
t_{0} & t_{1} & t_{2} \\
t_{0,1} & t_{1,1} & t_{2,1} \\
t_{0,2} & t_{1,2} & t_{2,2}
\end{array} z_{z_{2}}^{1}=\left[\begin{array}{ccc}
0.4461757 & -0.01239930 & -0.1460187 \\
-0.2262004 & -0.05189330 & 0.06557223 \\
0.4427031 & 0.01803564 & -0.1734312
\end{array}\right]\right.
\end{aligned}
$$

where $S$ is the diagonal matrix of all singular values of $H_{T^{*}}^{\bar{B}_{1}, \bar{B}_{2}}$ arranged in a decreasing order, $U$ is an unitary matrix whose columns are the left singular vectors of $H_{T^{*}}^{\bar{B}_{1}, \bar{B}_{2}}, V$ is an unitary matrix whose columns 
are the right singular vectors of $H_{T^{*}}^{\bar{B}_{1}, \bar{B}_{2}}$. We denote by $U^{\mathrm{H}}$ the Hermitian transpose of $U$ and $\bar{V}$ the conjugate of $V$.

Let $v_{i}=\left[v_{\alpha, i}\right]_{\alpha \in \bar{B}_{1}}$ and $w_{j}=\left[w_{\beta, j}\right]_{\beta \in \bar{B}_{2}}$ be respectively the $i^{\text {th }}$ and $j^{\text {th }}$ columns of $U^{\mathrm{H}}$ and $\bar{V}$. We denote by $v_{i}(\mathbf{x})=v_{i}^{\top} U_{r}^{\mathrm{H}}$ and $w_{j}(\mathbf{z})=w_{j}^{\top} \bar{V}_{r}$ the corresponding polynomials. The bases formed by these first $r$ polynomials are denoted $U_{r}^{\mathrm{H}}:=\left(v_{i}(\mathbf{x})\right)_{i=1, \ldots, r}$ and $\bar{V}_{r}:=\left(w_{j}(\mathbf{z})\right)_{j=1, \ldots, r}$. We will also denote by $U_{r}^{\mathrm{H}}\left(\operatorname{resp} . \bar{V}_{r}\right)$ the corresponding coefficient matrix, formed by the first rows (resp. columns) of $U^{\mathrm{H}}$ (resp. $\bar{V}$ ). We denote by $S_{r}$ the diagonal matrix of the first $r$ rows and columns of $S$, formed by the first $r$ singular values. To compute the multiplication matrices $M_{y_{1}}^{\bar{V}_{r}}$ and $M_{y_{2}}^{\bar{V}_{r}}$ we need to compute the following matrices

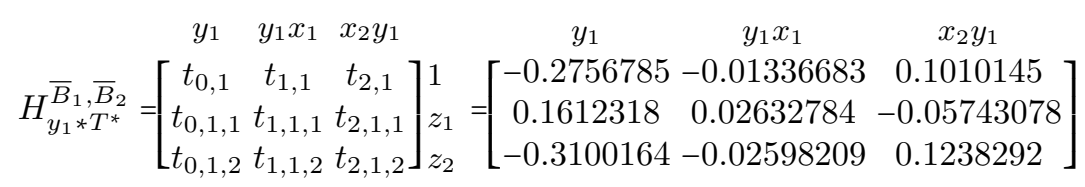

$$
\begin{aligned}
& H_{y_{2} * T^{*}}^{\bar{B}_{1}, \bar{B}_{2}}=\left[\begin{array}{ccc}
y_{2} & y_{2} x_{1} & x_{2} y_{2} \\
t_{0,2} & t_{1,2} & t_{2,2} \\
t_{0,2,1} & t_{1,2,1} & t_{2,2,1} \\
t_{0,2,2} & t_{1,2,2} & t_{2,2,2}
\end{array}\right]_{z_{2}}^{1} z_{1}=\left[\begin{array}{ccc}
-0.1209490 & -0.3195263 & -0.1485221 \\
0.1465160 & 0.09311605 & 0.03231415 \\
-0.1169341 & -0.1116610 & -0.00376925
\end{array}\right]
\end{aligned}
$$

Then we compute $M_{y_{1}}^{\bar{V}_{r}}=S_{r}^{-1} U_{r}^{\mathrm{H}} H_{y_{1} * T^{*}}^{\bar{B}_{1}, \bar{B}_{2}} \bar{V}_{r}$ and $M_{y_{2}}^{\bar{V}_{r}}=S_{r}^{-1} U_{r}^{\mathrm{H}} H_{y_{2} * T^{*}}^{\bar{B}_{1}, \bar{B}_{2}} \bar{V}_{r}$, and the eigenvectors $\mathbf{v}_{p}$ of $\sum_{j=1}^{2} l_{j} M_{y_{j}}^{\bar{V}_{r}}$ such that $\left|l_{j}\right| \leq 1, j=1, \ldots, 2$. To recover the points $\mathbf{u}_{p} \in \mathbb{C}^{n * k}$ for $p=1, \ldots, r$ of the form

$$
\mathbf{u}_{p}=\left[\begin{array}{c}
u_{p, 1,1} \\
u_{p, 1,2} \\
u_{p, 2,1} \\
u_{p, 2,2} \\
u_{p, 3,1} \\
u_{p, 3,2}
\end{array}\right]^{x_{1}} y_{2}
$$

We do the following:

In general we have $M_{x_{j, i_{j}}}^{\bar{V}_{r}} \mathbf{v}_{i}=u_{i, j, i_{j}} \mathbf{v}_{i}$, for $i=1, \ldots, r, j=1, \ldots, k, i_{j}=1,, n_{j}$ so in this case we get $M_{y_{1}}^{\bar{V}_{r}} \mathbf{v}_{p}=u_{p, 2,1} \mathbf{v}_{p}$ and $M_{y_{2}}^{\bar{V}_{r}} \mathbf{v}_{p}=u_{p, 2,2} \mathbf{v}_{p}$ for $p=1, \ldots, 3$ so we compute $u_{p, 2,1}$ and $u_{p, 2,2}$ for $p=1, \ldots, 3$. So that we get

$$
\begin{aligned}
& u_{p, 2,1}=[-0.746329870878-0.293761776025-0.304898408788] \\
& u_{p, 2,2}=[1.40328849510-0.336304368405-3.59031087599] .
\end{aligned}
$$

The eigenvectors $\mathbf{v}_{p} \in\left\langle 1, x_{1}, x_{2}\right\rangle$ for $p=1, \ldots, 3$ are up to a scalar the interpolation polynomials at the roots so that if the dual of the tensor has an affine decomposition $\underline{T}^{*}(\underline{\mathbf{x}}, \underline{\mathbf{y}}, \underline{\mathbf{z}})=\sum_{p=1}^{r} \omega_{p} \mathbf{e}_{\mathbf{u}_{p}}(\underline{\mathbf{x}}, \underline{\mathbf{y}}, \underline{\mathbf{z}})$ then $\underline{T}^{*}\left(\mathbf{v}_{p}\right)=$ $\sum_{p=1}^{r} \omega_{p} \mathbf{e}_{\mathbf{u}_{p}}\left(\mathbf{v}_{p}\right)=\lambda_{p} \omega_{p}, \underline{T}^{*}\left(z_{1} \mathbf{v}_{p}\right)=\sum_{p=1}^{r} \omega_{p} \mathbf{e}_{\mathbf{u}_{p}}\left(z_{1} \mathbf{v}_{p}\right)=\lambda_{p} \omega_{p} u_{p, 3,1}$ and $\underline{T}^{*}\left(z_{2} \mathbf{v}_{p}\right)=\sum_{p=1}^{r} \omega_{p} \mathbf{e}_{\mathbf{u}_{p}}\left(z_{2} \mathbf{v}_{p}\right)=$ $\lambda_{p} \omega_{p} u_{p, 3,2}$, for $p=1, \ldots, 3$. Then the values of $u_{p, 3,1}$ and $u_{p, 3,2}$ for $p=1, \ldots, 3$ come from the computation of the matrix:

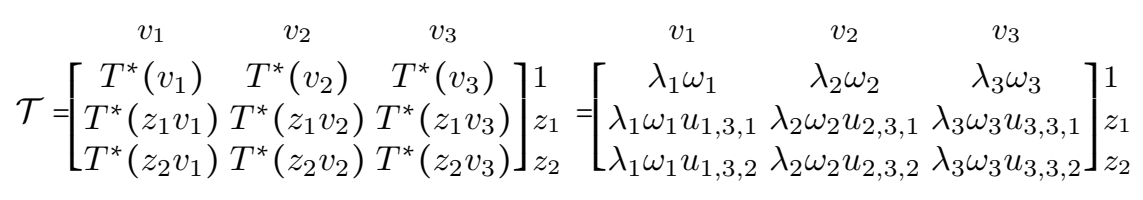


Therefore the value of $u_{p, 3,1}$ (resp. $u_{p, 3,2}$ ) comes from the ratio of the second row (resp. the third row) and the first row of the matrix for $p=1, \ldots, 3$. So that we get

$$
\begin{aligned}
& u_{p, 3,1}=\left[\begin{array}{lll}
-0.655842579065 & 0.0321233423462 & -0.520955291
\end{array}\right] \\
& u_{p, 3,2}=\left[\begin{array}{lll}
1.24749588143 & 0.403506877499 & 0.242728128570
\end{array}\right]
\end{aligned}
$$

The common eigenvectors of all $\left(M_{y_{j}}^{\bar{V}_{r}}\right)^{\top}$-are up to scalar- the evaluations, they are represented by vectors of the form $\mathbf{v}_{p}^{*}=\mu_{p}\left[1, u_{p, 1,1}, u_{p, 1,2}\right]$ in the dual basis of $\bar{B}_{1}=\left\langle 1, x_{1}, x_{2}\right\rangle$ then the computation of the coordinates of $u_{p, 1,1}$ and $u_{p, 1,2}$ come from the eigenvectors of the transpose of multiplication operators which are obtained by transposing the inverse of the matrix $V$ of vectors of $M_{y_{j}}^{\bar{V}_{r}}$ for $j=1, \ldots, 2$, therefore the value of $u_{p, 1,1}$ (resp. $u_{p, 1,2}$ ) comes from the ratio of the second element of $\mathbf{v}_{p}^{*}$ (resp. the third element) and the first element of it, so that

$$
\begin{aligned}
& u_{p, 1,1}=\left[\begin{array}{lll}
0.114279629148-1.08600705528 & 1.23814628617
\end{array}\right] \\
& u_{p, 1,2}=\left[\begin{array}{ll}
-0.405714894278-0.567603220082 & 0.873482418287
\end{array}\right] .
\end{aligned}
$$

Notice that the computation of $\omega_{p}, p=1, \ldots, 3$ can be done using the following formula $\omega_{p}=\frac{\left\langle T^{*} \mid \mathbf{v}_{p}\right\rangle}{\mathbf{v}_{p}\left(\mathbf{u}_{p}\right)}$ since if $\mathbf{v}_{p} \in\left\langle 1, x_{1}, x_{2}\right\rangle$ then $\mathbf{v}_{p}=a_{p}+x_{1} b_{p}+x_{2} c_{p}$ and $\mathbf{v}_{p}^{*}=\mu_{p}\left[1, u_{p, 1,1}, u_{p, 1,2}\right] \in\left(\left\langle 1, x_{1}, x_{2}\right\rangle\right)^{*}$, so that $\mathbf{v}_{p}\left(\mathbf{u}_{p}\right)=$ $a_{p}+u_{p, 1,1} b_{p}+u_{p, 1,2} c_{p}=\left\langle\mathbf{v}_{p} \mid \mathbf{v}_{p}^{*}\right\rangle$, the computation gives

$$
\omega=\left(\omega_{p}\right)_{1 \leq p \leq r}=[0.3185797522460 .088973893603120 .0386220875736]
$$

\section{References}

1. Anandkumar, A., Ge, R., Hsu, D., Kakade, S.M., Telgarsky, M. Tensor Decompositions for Learning Latent Variable Models (A Survey for ALT), pages 19-38. Springer International Publishing, Cham, 2015.

2. Bernardi, A., Daleo, N.S., Hauenstein, J.D., Mourrain, B.. Tensor decomposition and homotopy continuation. Differential Geometry and its Applications, August 2017.

3. Bernardi, A., Brachat, J., Comon, P., Mourrain, B. General tensor decomposition, moment matrices and applications. Journal of Symbolic Computation, 52:51-71, 2013.

4. Brachat, J., Comon, P., Mourrain, B., Tsigaridas, E.P. Symmetric tensor decomposition. Linear Algebra and Applications, 433(11-12):1851-1872, 2010.

5. Comon, P., Jutten, C. Handbook of Blind Source Separation: Independent component analysis and applications. Academic press, 2010.

6. Elkadi, M., Mourrain, B. Introduction à la résolution des systèmes polynomiaux, volume 59 of Mathématiques et Applications. Springer, 2007.

7. Harmouch, J., Khalil, H., Mourrain, B. Structured low rank decomposition of multivariate hankel matrices. Linear Algebra Appl., 2017. https://doi.org/10.1016/j.laa.2017.04.015

8. Connelly, A., Tournier, J.D., Calamante, F. Robust determination of the fiber orientation distribution in diffusion mri: Non-negativity constrained superresolved spherical deconvolution. NI, 35(4):1459--1472, 2007.

9. Jiang, T., Sidiropoulos, N.D.. Kruskal's permutation lemma and the identification of candecomp/parafac and bilinear models with constant modulus constraints. IEEE Transactions on Signal Processing, 52(9):2625-2636, Sept 2004 .

10. Landsberg, J.M. Tensors: Geometry and Applications. Graduate studies in mathematics. American Mathematical Soc., 2011.

11. De Lathauwer, L., Castaing, J. Tensor-based techniques for the blind separation of ds-cdma signals. Signal Processing, 87(2):322 - 336, 2007. Tensor Signal Processing.

12. Megherbi, T., Kachouane, M., Boumghar, F.O., Deriche, R. Détection des croisements de fibre en IRM de diffusion par décomposition de tenseur : Approche analytique. In Reconnaissance de Formes et Intelligence Artificielle (RFIA) 2014, France, June 2014.

13. Mourrain, B. Isolated points, duality and residues. J. of Pure and Applied Algebra, 117\&118:469-493, 1996.

14. Mourrain, B. Polynomial-exponential decomposition from moments. Found. Comput. Math., September 2016. https://doi.org/10.1007/s10208-017-9372-x 
15. Roch, S. A short proof that phylogenetic tree reconstruction by maximum likelihood is hard. IEEE/ACM Trans. Comput. Biol. Bioinformatics, 3(1), 2006.

16. Sanchez, E., Kowalski, B.R.. Tensorial resolution: A direct trilinear decomposition. Journal of Chemometrics, $4(1): 29-45,1990$.

17. Sidiropoulos, N.D., Giannakis, G.B., Bro, R. Blind parafac receivers for ds-cdma systems. IEEE Transactions on Signal Processing, 48(3):810-823, Mar 2000.

18. Smilde, A., Bro, R., Geladi, P. Multi-way analysis: applications in the chemical sciences. John Wiley \& Sons, 2005.

19. Atkins, M.S., Weldeselassie, T.Y., Barmpoutis, A. Symmetric positive semi-definite cartesian tensor fiber orientation distributions (ct-fod). Elsevier BV, Medical Image Analysis Journal, 16(6):1121-1129, 2012. 